\title{
Soliton interactions and transformations in colloidal media
}

\author{
Michał Matuszewski, ${ }^{1}$ Wieslaw Krolikowski, ${ }^{2}$ and Yuri S. Kivshar ${ }^{1}$ \\ ${ }^{1}$ Nonlinear Physics Center, Research School of Physics and Engineering, Australian National University, Canberra ACT 0200, Australia \\ ${ }^{2}$ Laser Physics Center, Research School of Physics and Engineering, Australian National University, \\ Canberra ACT 0200, Australia \\ (Received 29 September 2008; revised manuscript received 17 November 2008; published 11 February 2009)
}

\begin{abstract}
We study nonlinear light propagation in colloidal suspensions of spherical dielectric nanoparticles. We analyze the existence and properties of one-dimensional self-trapped beams (spatial optical solitons) in such media and demonstrate the existence of a bistability regime. The solitons corresponding to the two bistable branches have very different properties, and they can be easily distinguished by the measurement of the soliton width. We find that both types of solitons can form spontaneously through spatial modulational instability of continuous wave beams, but the solitons corresponding to the upper branch are more robust. This is also confirmed by the study of soliton collisions, where we describe a number of possible scenarios, including soliton amalgamation, destruction, reflection, deflection, and switching to another branch. We also find that the interaction of two mutually coherent solitons corresponding to different branches is phase independent and always repulsive. We provide a simple physical explanation of this phenomenon.
\end{abstract}

DOI: 10.1103/PhysRevA.79.023814

PACS number(s): 42.65.Tg, 42.65.Jx, 47.57.- $\mathrm{s}$

\section{INTRODUCTION}

Spatial optical solitons are formed when a change of the nonlinear refractive index induces an effective lensing effect that balances diffraction of the laser beam [1]. When an optical beam passes through a colloidal medium composed of a liquid suspension of dielectric nanoparticles, the optical gradient force acts against particle diffusion, increasing the refractive index in regions of higher light intensity. The corresponding local change of the refractive index is of the selffocusing type, and it allows for creation of spatial optical solitons in the form of self-trapped optical beams, as was demonstrated in both theoretical and experimental studies [2-5]. Recently, it was shown theoretically [5] that the optical response of a colloidal medium in the hard-sphere approximation can lead to optical bistability and the existence of two stable soliton solutions for the same beam poweri.e., soliton bistability of the first kind [6,7]. This opens novel opportunities for the control of soliton beams via their collision and switching.

The interaction of optical solitons is a problem of fundamental importance, and it has been studied thoroughly in the past decades $[1,8]$. While collisions of solitons described by integrable models (e.g., for one-dimensional Kerr solitons) are always elastic, those of nonintegrable models (including bistable) demonstrate the richness of the dynamics depending on the detailed form of the nonlinearity [9]. For instance, soliton annihilation or amalgamation of two solitons from the lower branch into one upper-branch soliton was predicted [10].

In this paper, we study collisions of bistable solitons in the model of colloidal media and demonstrate that the solitons can undergo transformations during their interactions. Moreover, we reveal that the interaction of two solitons from different branches is phase independent and always repulsive, a surprising result in view of all previous studies $[1,8]$. We provide a simple physical explanation of this phenomenon.

\section{MODEL}

The model of nonlinear laser beam propagation in a colloidal suspension of dielectric hard spheres was described in [5]. We assume that the refractive index of colloidal particles, $n_{p}$, is slightly higher than the background index $n_{b}$ and that the particle diameter is much smaller than the laser wavelength in the background medium, $d \ll \lambda_{0} / n_{b}$ (Rayleigh regime). We assume that the dielectric colloidal particles interact with each other through a hard-sphere potential. In the steady state the colloidal particles satisfy the Maxwellian velocity distribution, which follows from the phase-space density in the canonical ensemble $\varrho \sim \exp \left(-E / k_{\mathrm{B}} T\right)$. The pressure exerted by colloidal particles can be obtained from the equation of state in analogy with the hard-sphere gas [11]:

$$
\frac{\beta p}{\rho}=Z(\eta)
$$

where $\beta=1 / k_{\mathrm{B}} T, p$ is the pressure, $\rho$ is the colloidal particle density, $Z(\eta)$ is the compressibility, and $\eta=\rho / \rho_{0}$ is the packing fraction. In the case of ideal gas, we have $Z=1$. For a hard-sphere gas, the Carnahan-Starling formula $Z \approx(1+\eta$ $\left.+\eta^{2}-\eta^{3}\right) /(1-\eta)^{3}$ gives a very good approximation up to the fluid-solid transition at $\eta \approx 0.5$ [11]. This phenomenological formula is in agreement with exact perturbation theory calculations as well as molecular-dynamics simulations.

In the presence of a slowly varying external potential, such as that induced by the presence of optical beam, the particle velocity distribution is locally Maxwellian. The gradient of the density $\rho(\mathbf{r})$ is assumed to be locally parallel to $\hat{x}$, and we consider a small box of volume $d V=d x d S$, with length $d x$ and normal surface $d S$. The difference in pressure exerted on the right and left surfaces, $d p$, gives rise to an effective force acting on the colloidal particles, $F_{\text {int }}$. It is equal to the external force that is necessary to sustain the density gradient, and $d p=-F_{\text {int }} / d S=-f_{\text {int }} \rho d V / d S=-f_{\text {int }} \rho d x$, where $f_{\text {int }}$ is the average force acting on a single particle. 
Using Eq. (1), we get $d(\rho Z) / d x=-f_{\text {int }} \rho \beta$. The particle current density is equal to

$$
\vec{j}=\rho \mu\left(\vec{f}_{\mathrm{ex}}+\vec{f}_{\text {int }}\right)=\rho \mu \vec{f}_{\mathrm{ex}}-D \vec{\nabla}(\rho Z),
$$

where $\mu$ is the particle mobility and $D=\mu / \beta$ is the diffusion constant. In the ideal gas limit, this equation becomes Eq. (3) of [4]. Let $m=n_{\mathrm{p}} / n_{\mathrm{b}}$ be the ratio of the colloidal particle refractive index to the background refractive index. The polarizability of a sphere is given by

$$
\alpha=3 V_{\mathrm{p}} \varepsilon_{0} n_{\mathrm{b}}^{2} \delta,
$$

where $V_{\mathrm{p}}$ is the sphere volume and $\delta=\left(m^{2}-1\right) /\left(m^{2}+2\right)$. If we look for the steady state $(\vec{j}=\overrightarrow{0})$ in the presence of an optical field gradient $\left[\vec{f}_{\mathrm{ex}}=(\alpha / 4) \vec{\nabla} I\right.$, where $\left.I=|E|^{2}\right]$, we obtain

$$
\rho \frac{\alpha \beta}{4} \frac{d I}{d x}=\frac{d(\rho Z)}{d x},
$$

which can be solved analytically to give the dependence $I(\eta)$ :

$$
\frac{\alpha \beta}{4} I(\eta)=g(\eta)-g\left(\eta_{0}\right),
$$

where $g(\eta)=(3-\eta) /(1-\eta)^{3}+\ln \eta$ and $\eta_{0}$ is the background packing fraction.

Assuming a relatively low packing fraction, the corresponding nonlinear refractive index change can be approximately calculated using the Maxwell-Garnett formula [12]

$$
\varepsilon_{\text {eff }}=\varepsilon_{\mathrm{b}}+\frac{3 \eta \varepsilon_{\mathrm{b}}\left(\varepsilon_{\mathrm{p}}-\varepsilon_{\mathrm{b}}\right)}{\left[\varepsilon_{\mathrm{p}}+2 \varepsilon_{\mathrm{b}}-\eta\left(\varepsilon_{\mathrm{p}}-\varepsilon_{\mathrm{b}}\right)\right]} .
$$

For low refractive index contrast $\left(\varepsilon_{\mathrm{p}} / \varepsilon_{\mathrm{b}} \approx 1\right)$, we have

$$
\varepsilon_{\mathrm{eff}} \approx \varepsilon_{\mathrm{b}}+\frac{3 \varepsilon_{\mathrm{b}}\left(\varepsilon_{\mathrm{p}}-\varepsilon_{\mathrm{b}}\right)}{\left(\varepsilon_{\mathrm{p}}+2 \varepsilon_{\mathrm{b}}\right)} \eta=\varepsilon_{\mathrm{b}}(1+3 \delta \eta) .
$$

Substituting this formula to the Helmholtz equation $\nabla^{2} E$ $+k_{0}^{2} n_{\mathrm{eff}}^{2} E=0$, we obtain the propagation equation for a slowly varying envelope of electric field $u(\widetilde{\mathbf{r}})$ defined by $E(\widetilde{\mathbf{r}})$ $=(2 / \sqrt{\alpha \beta}) u(\widetilde{\mathbf{r}}) \exp \left[i k\left(1+3 \delta \eta_{0}\right)^{1 / 2} \tilde{z}\right]$ :

$$
i \frac{\partial u}{\partial \widetilde{z}}+\frac{1}{2 k \sqrt{1+3 \delta \eta_{0}}}\left[\frac{1}{2} \nabla_{\widetilde{\perp}}^{2} u+3 k^{2} \delta\left(\eta-\eta_{0}\right) u\right]+i \frac{\gamma}{2} u=0,
$$

where $k=2 \pi n_{b} / \lambda_{0}$ and the additional last term on the lefthand side accounts for damping due to Rayleigh scattering from the dielectric spheres. The damping coefficient is given by $\gamma=2 \pi^{5} \rho \delta^{2} d^{6} /\left(3 \lambda^{4}\right)$ [13], where $\lambda=\lambda_{0} / n_{b}$. Additionally, for steady-state solutions, relation (5) gives

$$
|u|^{2}=g(\eta)-g\left(\eta_{0}\right)
$$

at each point in space, where $g(\eta)=(3-\eta) /(1-\eta)^{3}+\ln \eta$. The typical dependence $\eta\left(|u|^{2}\right)$ in this case is shown in Fig. 1. In the low-intensity limit, the nonlinear index change is Kerr-like (proportional to intensity). For higher intensities, it is well described by the exponential model of $[3,4]$. Finally,

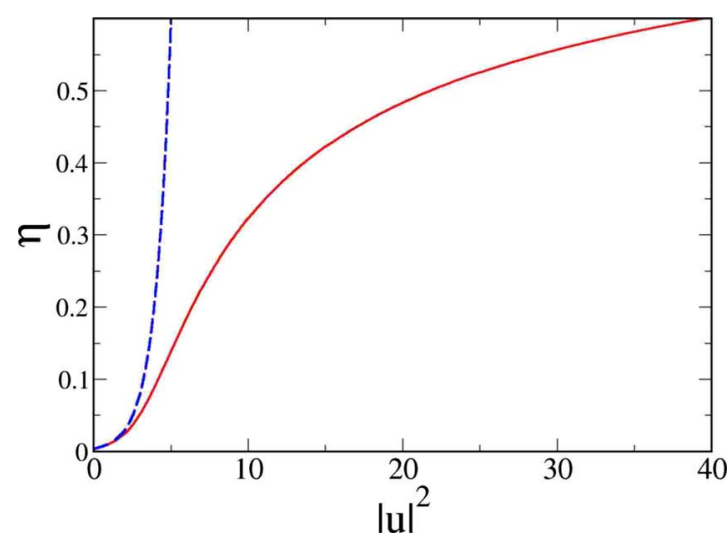

FIG. 1. (Color online) Packing fraction $\eta$ of colloidal particles vs the light intensity (solid line). The dashed line shows the dependence of the exponential model.

for higher densities the particle hard-sphere interactions become significant and the nonlinearity saturates as the exponential model breaks down.

We renormalize the spatial coordinates according to $(x, y)=\left(2 / 3 k^{2} \delta\right)^{1 / 2}(\widetilde{x}, \widetilde{y})$ and $z=\left[2\left(1+3 \delta \eta_{0}\right)^{1 / 2} / 3 k \delta\right] \widetilde{z}$, obtaining

$$
i \frac{\partial u}{\partial z}+\frac{1}{2} \nabla_{\perp}^{2} u+\left(\eta-\eta_{0}\right) u+i \frac{\Gamma}{2} \eta u=0,
$$

where the renormalized damping coefficient is

$$
\Gamma=\frac{2}{3} \pi^{3} \sqrt{1+3 \delta \eta_{0}}\left(\frac{d}{\lambda}\right)^{3} \delta .
$$

From Eq. (10) and formula (11), we conclude that the effect of scattering losses depends strongly on the ratio of the particle size to the laser wavelength. In the following, we will ignore the effect of damping in accordance with the assumption $d / \lambda \ll 1$. Later on we will verify correctness of this assumption.

\section{MODULATIONAL INSTABILITY}

We consider stability of continuous wave solutions of Eq. (10) with background packing fraction $\eta_{0}=10^{-3}$ in the form $u=\left|u_{s}\right| \exp \left(i \eta_{s} z+i \varphi\right)$, where relation (9) holds between $u_{s}$ and $\eta_{s}$. The initial stage of modulational instability was studied analytically in Ref. [5], with a general conclusion that the beams are always unstable. Here we investigate in detail the subsequent beam evolution and the formation of solitons.

A typical example of propagation of an initially homogeneous beam is presented in Fig. 2. The initial beam is perturbed by a Gaussian noise equal to $\sigma=1 \%$ of the beam intensity to reflect the experimental beam distortion and impurities, $u(x, z=0)=u_{s}[1+f(x, \sigma)]$, where the value of $f(x, \sigma)$ is chosen independently at each grid point according to a random Gaussian distribution. We find that, depending on the beam intensity, the beam can undergo modulation instability, which leads to filamentation into large-scale solitons corresponding to the lower stable branch (see below), shown in Fig. 2(a), or small-scale upper branch solitons, as shown in 

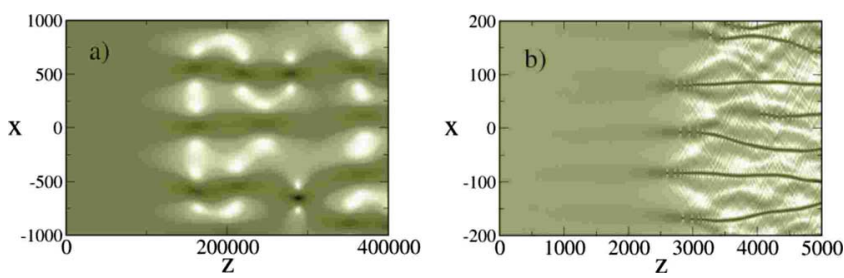

FIG. 2. (Color online) Modulation instability leading to the formation of solitons from the (a) lower branch for $\left|u_{0}\right|^{2}=0.05$ and (b) upper branch for $\left|u_{0}\right|^{2}=1$. The threshold value separating these two scenarios is $\left|u_{0}\right|^{2} \approx 0.08$.

Fig. 2(b). The second scenario occurs for the beam intensity over the threshold $\left|u_{0}\right|^{2} \approx 0.08$. While the lower branch solitons can merge, disappear, and reappear, the upper-branch solitons display more robustness and are well distinguishable. For the scenario presented in Fig. 2(a), the scattering losses prevent the formation of solitons for particle diameter to wavelength ratios larger than $d / \lambda \sim 10^{-2}$ due to the long propagation distance.

\section{SOLITONS}

In this section, we study one-dimensional spatial solitons and look for localized solutions of Eq. (10) in the form $u(\mathbf{r})=A(x) \exp (i \kappa z)$. In this case, the propagation equation (10) reduces to

$$
-\kappa A+\frac{1}{2} \frac{d^{2} A}{d x^{2}}+\left(\eta-\eta_{0}\right) A=0
$$

and the soliton profiles can be obtained numerically [5]. In Fig. 3(a) we show the dependence of the soliton width $W$ $=3 \int|x||u|^{2} d x$ and power $P=\int|u|^{2} d x$ versus the propagation constant $\kappa$. The two stable branches with a positive slope $d P / d \kappa>0$ are separated by an unstable branch with a negative slope [6]. Bistable solutions exist within the power range $P \approx 33-51$. These solitons fulfill all the three stability con- ditions required for robustness during collisions [9].

Example of bistable soliton profiles for $P=40$ are presented in Figs. 3(b) and 3(c). The width of the soliton from the lower branch is approximately 20 times larger than the width of the soliton from the upper branch carrying the same beam power. Therefore, these solitons can be easily distinguished in experiment by measuring their width. For the experimental parameters $\lambda=1064 \mathrm{~nm}$, particle diameter $d$ $=30 \mathrm{~nm}, n_{p}=1.56$, and $n_{b}=1.33$ (polystyrene beads in water), the total soliton beam power is about $2 \mathrm{~W}$ and the peak intensity of the soliton from the upper branch reaches $\approx 300 \mathrm{MW} / \mathrm{cm}^{2}$.

\section{SOLITON INTERACTIONS}

Now we study numerically collisions of mutually coherent solitons from the two bistable branches. We employ different configurations and collision parameters. The initial state is selected in the form of two stationary soliton solutions $u_{1}(x)$ and $u_{2}(x)$ separated by a distance $2 x_{0}$ large compared to each of the soliton widths. The solitons have imprinted opposite linear phases $k_{0}$, which resemble the initial beam tilt, and a constant phase difference $\Delta \varphi$ :

$$
u(x, z=0)=u_{1}\left(x+x_{0}\right) e^{i k_{0} x}+u_{2}\left(x-x_{0}\right) e^{-i k_{0} x+i \Delta \varphi} .
$$

Our numerical studies reveal that interaction of two solitons from the same branch and from different branches are dramatically different, and we consider them separately in the following study of the possible collision outcomes. We notice that in all cases presented below, the two interacting solitons carry the same power. We also perform numerical simulations involving collisions of solitons with different powers, but these did not lead to any qualitatively different scenario. For the values of the experimental parameters proposed in Sec. IV, the damping coefficient is calculated as $\Gamma$ $=5 \times 10^{-5}$. Therefore, the soliton power loss due to the Rayleigh scattering in most of the cases is negligible, except for the longest simulations where for upper branch solitons it can reach $10 \%$.


FIG. 3. (Color online) (a) Soliton power and width vs the propagation constant $\kappa$ for $\eta_{0}=10^{-3}$. Bottom panels show the soliton intensity profile (solid) and colloidal particle packing fraction (dashed) for bistable solitons carrying power $P=40$ from (b) the lower stable branch and (c) the upper stable branch. Notice the difference in the width scale. 

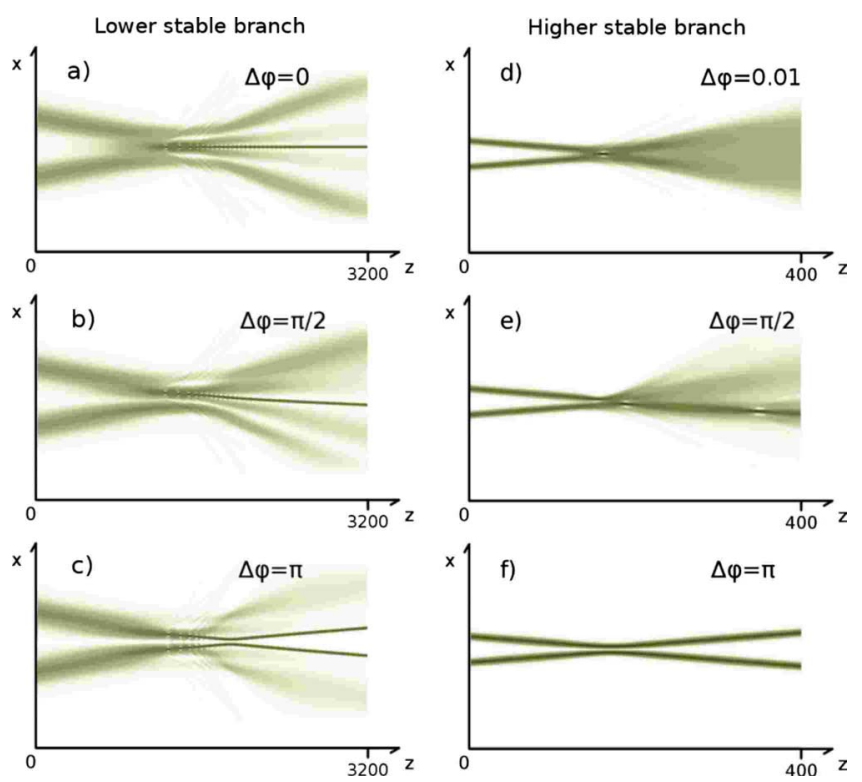

FIG. 4. (Color online) (a)-(c) Collisions of solitons from the lower stable branch for $P=45, k_{0}=0.05$, and different values of the phase difference $\Delta \varphi$. In panels (d), (e), and (f) collisions of solitons from the higher branch are presented for $P=33$ and $k_{0}=0.1$.

\section{A. Collisions of solitons from the same branch}

We consider collisions of two solitons from the lower branch as well as from the upper branch. We find that far from the instability threshold corresponding to the limit of a stable branch, the soliton interaction is similar to the previously described elastic collisions of Kerr solitons (for lower branch) or inelastic collisions in a saturable medium (upper branch) [1]. This interaction can be either repulsive or attractive depending on the phase difference $\Delta \varphi$, and it can also lead to a power transfer between solitons in nonintegrable case $[1,8]$.

However, if the soliton power $P$ or propagation constant $\kappa$ approaches the instability threshold, the soliton interaction can have a dramatically different character. In Figs. 4(a) and 4(b) we show the results for collisions of two high-power solitons from the lower branch, amalgamating into one soliton from the upper branch, with a significant amount of beam power being radiated. In the scenario from Fig. 4(c), the repulsive force between out-of-phase solitons causes them to switch to the upper branch. Figure 4(d) shows the annihilation of two solitons from the upper branch close to the instability threshold. This scenario does not occur for perfectly in-phase solitons, but a slight phase difference, unavoidable in experimental conditions, leads to destabilization and destruction of the soliton beams.

\section{B. Collisions of solitons from different branches}

In contrast to the case described above, the interaction of solitons from two different branches has a completely different character than soliton collisions in any model considered before [8]. The main feature is that, despite the fact that the beams are mutually coherent, their interaction appears to be phase independent; i.e., it does not depend on $\Delta \varphi$. At the
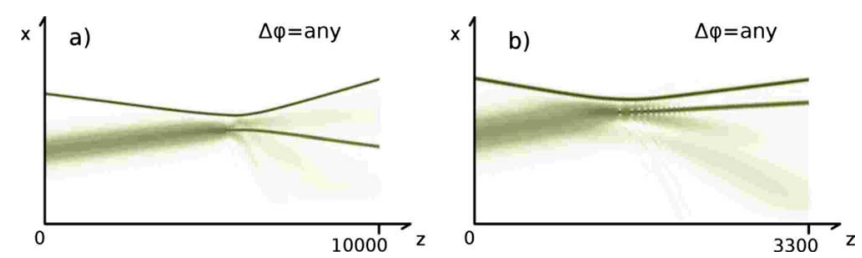

FIG. 5. (Color online) Switching from the lower to the upper branch triggered by collision with another soliton for soliton power $P=50$ and (a) $k_{0}=0.01$ or (b) $k_{0}=0.03$. Powers of both solitons are equal to $P$.

same time, the interaction is always repulsive, the effect being opposite to what is observed in models of mutually incoherent optical beams in self-focusing media [8].

In Figs. 5 and 6, we present some of the possible scenarios of soliton collisions. In Figs. 5(a) and 5(b), the soliton of the lower branch is triggered to switch to a soliton of the upper branch. In Fig. 6(a) solitons pass through each other as the initial beam tilt is too large for the interaction to occur. In Figs. 6(b) and 6(c) the soliton of the upper branch is deflected by the lower-branch soliton, which eventually diffracts, and in Fig. 6(d) both solitons undergo reflection. We notice that while both beams carry the same power, the upper-branch soliton is much more robust and appears in unchanged form after the collision, except for a possible change in the propagation angle. Figure 7 summarizes the results of a systematic study of these soliton collisions, with the regions in parameter space $\left(P, k_{0}\right)$ corresponding to the various scenarios described above.

\section{Explanation of the phase-independent repulsive soliton interaction}

As was pointed out above, the collisions of solitons belonging to different branches differ drastically from those involving solitons of the same branch. In a sharp contrast to all previously considered types of interaction between optical solitons $[1,8]$, the soliton collisions we described are phase
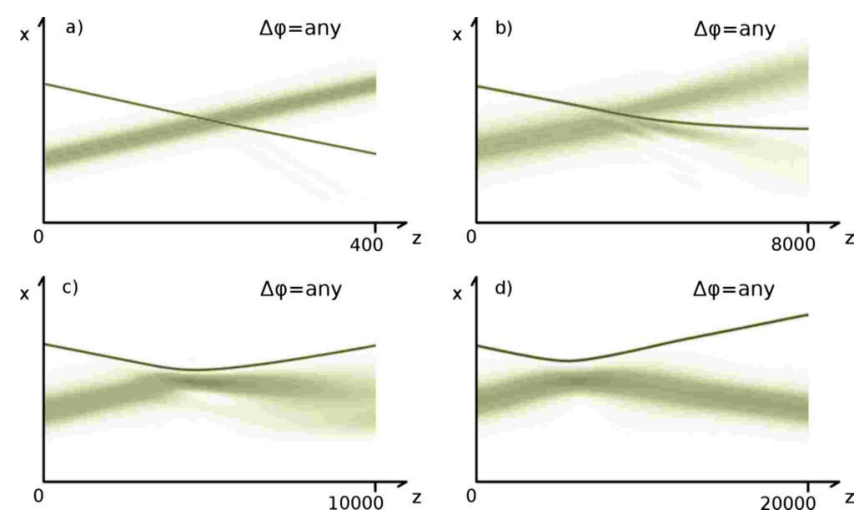

FIG. 6. (Color online) Other scenarios for collision of two solitons from different branches. For high transverse velocities, the solitons pass through each other as shown in (a) for $P=50$ and $k_{0}$ $=0.5$. For lower velocities, the interaction leads to (b) soliton deflection for $P=35$ and $k_{0}=0.025$, (c) destruction for $P=40$ and $k_{0}$ $=0.02$, or (d) reflection for $P=45$ and $k_{0}=0.01$. 


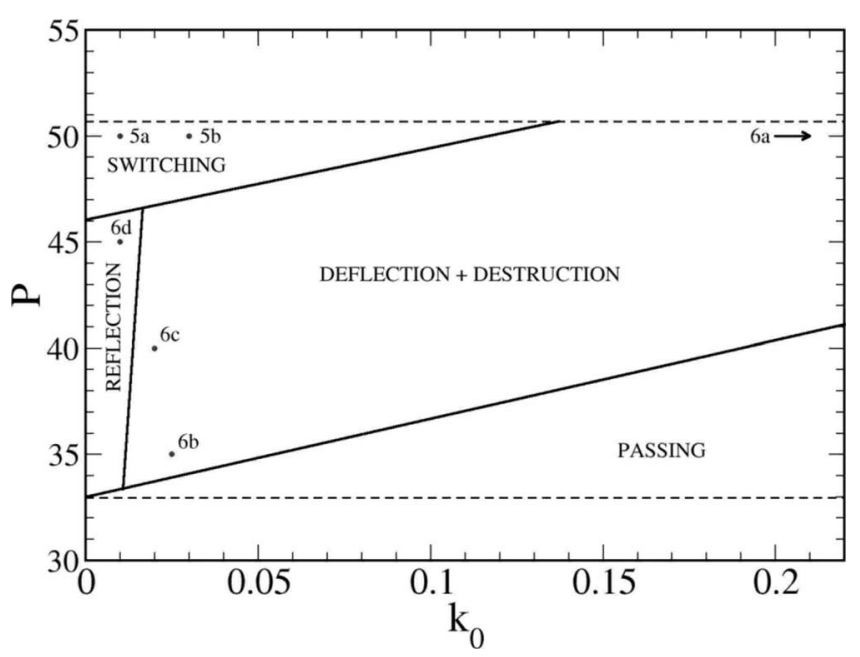

FIG. 7. Diagram of collision outcomes for two bistable solitons from different branches. The marked points correspond to numerical data from Figs. 5 and 6 . The central area corresponds to scenarios where the lower-branch soliton is destructed and the higherbranch soliton changes the propagation direction. The dashed lines determine the bistability region.

independent and always repulsive. Typically, the coherent interaction of solitons depends strongly on their relative phase, so that the collision is attractive for in-phase solitons and is repulsive for out-of-phase solitons. The property of phase independence revealed here can be explained by a strong mismatch in the propagation constants. From Fig. 3 it is evident that for solitons belonging to different branches their phase velocities, expressed by the propagation constant $\kappa$, differ by more than two orders of magnitude. This phase mismatch leads to very fast oscillations of the soliton mutual phase in the interaction area. As a result, the coherent contribution to the soliton interaction averages out and the interaction becomes effectively incoherent. As such, it can be described not by a single equation, but rather two incoherently coupled nonlinear equations for the two colliding solitons:

$$
\begin{aligned}
& i \frac{\partial \widetilde{u}_{1}}{\partial z}+\frac{1}{2} \nabla_{\perp}^{2} \widetilde{u}_{1}+\left(\eta-\eta_{0}\right) \widetilde{u}_{1}=0, \\
& i \frac{\partial \widetilde{u}_{2}}{\partial z}+\frac{1}{2} \nabla_{\perp}^{2} \widetilde{u}_{2}+\left(\eta-\eta_{0}\right) \widetilde{u}_{2}=0,
\end{aligned}
$$

where $\eta$ is determined by Eq. (9) with the light intensity being just a sum of the intensities of both the solitons, $|u|^{2}$ $=\left|\widetilde{u}_{1}\right|^{2}+\left|\widetilde{u}_{2}\right|^{2}$.
To confirm this finding, we solve Eqs. (14) numerically and compare the profile of the total intensity $|u|^{2}$ to that obtained in the case of the coherent interaction, as plotted in Figs. 4-6. Indeed, we observe that the outcome of the interaction is identical in both cases, with the only visible difference being the disappearance of interference fringes in the area of the soliton overlap in the incoherent case. Moreover, we find that there is no exchange of soliton power, so each of the solitons remains in its initial component.

Presently, it is commonly believed that the incoherent interaction of solitons results always in their attraction, but not repulsion $[1,8]$. In fact, each of the solitons "feels" the other one as an effective attractive potential; hence, the soliton coalescence or passing can be anticipated. However, a soliton reflection from a narrow, deep, attractive potential wells or defects has been already reported for different systems $[14,15]$. It was shown that if the soliton velocity falls below a certain threshold, a repulsive interaction between the soliton and an attractive potential can occur. In our case, the conditions required for this phenomenon to take place correspond well to the parameters of the soliton interactions. The narrow but intense soliton from the upper branch plays the role of an effective defect potential for a broad but weak soliton from the lower branch, which is reflected if its kinetic energy (velocity) is low enough; see Fig. 7. We present here evidence of a phase-independent repulsive interaction of solitons.

\section{CONCLUSION}

We have studied numerically bistable spatial optical solitons and their collisions in colloidal media. We have revealed a number of various scenarios of the soliton interaction, including such effects as amalgamation, destruction, reflection, deflection, and switching to another branch. We also found that the collision of two coherent solitons corresponding to different branches is effectively incoherent and always repulsive, and we have provided a simple physical explanation for this phenomenon.

\section{ACKNOWLEDGMENTS}

The authors acknowledge support of the Research School of Physics and Engineering of the Australian National University and the Australian Research Council.
[1] Y. S. Kivshar and G. P. Agrawal, Optical Solitons: From Fibers to Photonic Crystals (Academic Press, San Diego, 2003).

[2] A. Ashkin, J. M. Dziedzic, and P. W. Smith, Opt. Lett. 7, 276 (1982); P. J. Reece, E. M. Wright, and K. Dholakia, Phys. Rev. Lett. 98, 203902 (2007); C. Conti, G. Ruocco, and S. Trillo, ibid. 95, 183902 (2005).
[3] R. Gordon, J. T. Blakely, and D. Sinton, Phys. Rev. A 75, 055801 (2007).

[4] R. El-Ganainy, D. N. Christodoulides, C. Rotschild, and M. Segev, Opt. Express 15, 10207 (2007).

[5] M. Matuszewski, W. Krolikowski, and Y. S. Kivshar, Opt. Express 16, 1371 (2008). 
[6] A. E. Kaplan, Phys. Rev. Lett. 55, 1291 (1985).

[7] S. L. Eix and R. H. Enns, Phys. Rev. A 47, 5009 (1993).

[8] G. I. Stegeman and M. Segev, Science 286, 1518 (1999).

[9] R. H. Enns, S. S. Rangnekar, and A. E. Kaplan, Phys. Rev. A 36, 1270 (1987).

[10] R. H. Enns, D. E. Edmundson, S. S. Rangnekar, and A. E. Kaplan, Opt. Quantum Electron. 24, S1295 (1992).

[11] J.-P. Hansen and I. R. McDonald, Theory of Simple Liquids, 3rd ed. (Elsevier, Amsterdam, 2006).
[12] J. C. M. Garnett, Philos. Trans. R. Soc. London, Ser. A 203, 385 (1904).

[13] H. C. van de Hulst, Light Scattering by Small Particles (Dover, New York, 1981).

[14] Yu. S. Kivshar, Zhang Fei, and L. Vázquez, Phys. Rev. Lett. 67, 1177 (1991).

[15] R. H. Goodman, P. J. Holmes, and M. I. Weinstein, Physica D 192, 215 (2004); C. Lee and J. Brand, Europhys. Lett. 73, 321 (2006). 